\title{
SPECTROSCOPY AND 3D-CISS SEQUENCE IN CYSTIC LESIONS OF BRAIN: A PROSPECTIVE EVALUATION ON MRI
}

\author{
Mary Hazarika Bhuyan'1, R. K. Bhuyan ${ }^{2}$
}

${ }^{1}$ Associate Professor, Department of Radiology, Assam Medical College, Dibrugarh.

2 Professor, Department of Surgery, Assam Medical College, Dibrugarh.

\begin{abstract}
BACKGROUND

The present study was undertaken to differentiate between benign and malignant cystic lesions of the brain and to establish a diagnosis on MRI. To study the role of MR spectroscopy and 3D CISS in the evaluation of various cystic lesions in the brain wherever applicable.
\end{abstract}

\section{METHODS}

The study was conducted on 35 patients at Assam Medical College and Hospital, Dibrugarh, with a suspicion of brain SOLs by neurologists, paediatricians and physicians from in and around Dibrugarh over a period of one year from January 2011 to December 2012. Out of the 35 patients who were evaluated, Arachnoid cyst (20\%) was the most common non-neoplastic cystic lesion, Craniopharyngioma (5.7\%) was the most common benign neoplastic cystic lesion and Glioma (17.1\%) was the most common malignant neoplastic cystic lesion. In the present study, conventional MRI with additional techniques like MRS and 3D CISS together were found to be accurate in $94.28 \%$ of cases. One case of MRI diagnosis of low-grade glioma came out to be high-grade on HPE. One case of MRI diagnosis of cystic astrocytoma came out to be a benign vascular lesion-Haemangioma on HPE.

\section{CONCLUSION}

MRI is the most sensitive modality in the characterisation of intracranial cystic lesions. MRS is a complementary to conventional MRI in diagnosing the cystic lesions of the brain and influencing the overall management of these lesions; 3D-CISS sequence is superior and more accurate than routine SE sequences in the diagnosis of intraventricular cystic lesions.

\section{KEYWORDS}

3D CISS (3 Dimensional Constructive Interference in Steady State), MR Spectroscopy.

HOW TO CITE THIS ARTICLE: Bhuyan MH, Bhuyan RK. Spectroscopy and 3D-CISS sequence in cystic lesions of brain: a prospective evaluation on MRI. J. Evolution Med. Dent. Sci. 2016;5(74):5463-5469, DOI: 10.14260/jemds/2016/1237

\section{INTRODUCTION}

Cysts and cystic-appearing intracranial lesions are common findings with routine cerebral imaging examination. These lesions often represent a challenge in diagnosis. Intracranial cystic lesions have wide pathologic and imaging spectra of which some require an aggressive and tailored treatment, whereas many others remain asymptomatic and do not require followup or intervention. Intracranial cysts can be divided into non-neoplastic lesions that are often of developmental origin, but comprise of infectious cysts as well and neoplastic lesions that include benign cysts associated with low-grade tumours and cysts as a component of higher grade neoplasms.[1] Intracerebral cystic lesions can lead to a real diagnostic challenge for both the radiologist and the neurologist. In this respect, MRI diffusion-weighted sequences and MR spectroscopy proved to be particularly useful recently. However, these techniques are not widely available and therefore features seen with CT scans and classical MRI sequences are still important for the differential diagnosis.

Financial or Other, Competing Interest: None.

Submission 21-12-2015, Peer Review 26-12-2015,

Acceptance 28-12-2015, Published 14-09-2016.

Corresponding Author:

Dr. Mary Hazarika Bhuyan,

C/o. Dr. R. K. Bhuyan

PQ12, AMCH, Dibrugarh-786002.

E-mail: maryhazarikabhuyan@yahoo.com

DOI: $10.14260 /$ jemds/2016/1237
In particular, sometimes it may be difficult to distinguish between cerebral abscesses and cystic necrotic tumours based on imaging.

This aspect can be extremely important especially in lifethreatening cases when a correct diagnosis leads to a prompt treatment. Nevertheless, in unclear cases, biopsy or surgery and subsequent pathological analysis can establish the final precise diagnosis.[2]

MR spectroscopy is a potential tool for differential diagnosis between brain abscesses and non-infectious lesions such as primary brain tumour, lymphoma, brain metastasis and tuberculoma. MRS provides information about the possible extent and nature of changes on a routine MRI scan by analysing the presence and/or ratio of tissue metabolites such as NAA, creatinine, choline and lactate, etc. ${ }^{[3]}$ Threedimensional (3D) Constructive Interference in Steady State (CISS) is a gradient echo MRI sequence that is used to investigate a wide range of pathologies when routine MRI sequences do not provide the desired anatomic information.

The increased sensitivity of the 3D CISS sequence is an outcome of the accentuation of the $\mathrm{T} 2$ values between Cerebrospinal Fluid (CSF) and pathological structures. Apart from its well-recognised applications in the evaluation of the cranial nerves, CSF rhinorrhoea and aqueduct stenosis, CISS sequence to be useful for the cisternal spaces, cavernous sinuses and the ventricular system where it is useful for detecting subtle CSF-intensity lesions that maybe missed on routine spin-echo sequences. This information helps in the management of these conditions. [3] 


\section{MATERIAL AND METHODS}

With the aim to evaluate cystic lesion of brain with emphasis on spectroscopy and CISS on MRI, this study was conducted on 35 patients at Assam Medical College and Hospital, Dibrugarh, with a suspicion of brain SOLs by neurologists, paediatricians and physicians from in and around Dibrugarh over a period of one year from January 2011 to December 2012.

\section{Study Design}

This was a prospective study done to evaluate the efficacy of conventional MRI with special MRI techniques like MR spectroscopy and 3D CISS in the diagnosis of cystic lesions of brain and to differentiate benign and malignant cystic lesions. The study comprised of all the patients clinically suspected to be having brain SOLs and referred for MR evaluation to the Department of Radiodiagnosis, Assam Medical College and Hospital, Dibrugarh. Inclusion Criteria was all patients with clinically suspected brain SOLs and incidentally diagnosed cystic lesion by CT. Patients of all age groups were included irrespective of sex. Exclusion criterion were intraparenchymal granulomatous lesions (like intraparenchymal neurocysticercosis, tuberculomas, toxoplasmosis, etc.) and brain abscesses. Normal variant or developmental cystic lesions like enlarged Perivascular Spaces (PVSs), cavum septi pellucidi, cavum veli interpositi, cavum vergae, mega cisterna magna, Dandy-Walker malformation, choroidal fissure cysts, choroid plexus cysts and pineal cysts $(<1.5 \mathrm{~cm})$.

All patients with general contraindications to MRI like claustrophobia, metallic implants, pacemakers, etc. Paediatric patients who were not co-operative were sedated by giving oral/IV sedatives. Consent of the patient and attendant was taken for the contrast examination. The procedure and objectives of performing examination were explained to the patients. The patients enrolled to the study were subjected to MRI using SIEMENS MAGNETOM AVANTO 1.5 TESLA machine. Head coil was used in all the patients in supine position. Conventional spin echo sequences, axial T1, T2 and FLAIR; Coronal T2; Sagittal T1; axial GRE (Gradient Recalled Echo); DWI (Diffusion Weighted Imaging) followed by postcontrast axial, coronal and sagittal images were obtained. Additional sequence like 3D CISS[4] was acquired for extraparenchymal cystic lesions including both extra-axial and intraventricular group whenever required. Spectroscopy was avoided in small cystic lesions close proximity to the bone.

Spectral heights of metabolites like choline, creatinine, Nacetylaspartate, lactate, lipid and amino acids were studied. Metabolite ratios like $\mathrm{Cho} / \mathrm{Cr}$ and NAA/Cr were also studied. As reference standards values of $\mathrm{Cho} / \mathrm{Cr}>1.5$ and NAA/Cr $<1.6$ were taken as abnormal, which was similar to study conducted by N. Meena et al (2015).[5] All cases were followed up and clinically correlated to confirm the diagnosis given on MRI scan either by the characteristic imaging features or postoperative histopathological reports wherever possible. Descriptive statistical approach was used to describe patient's characteristics using numbers, combined accuracy of conventional MRI and additional techniques like MRS and 3D CISS was evaluated. All the statistical calculations were done using Microsoft Excel, 2010.

\section{Findings}

Maximum number of patients belonged to 20-29 age group, which comprised $25.71 \%$ of the whole study group followed

by $20 \%$ in 30-39 age group. Out of 35 cases, male accounts for 23 cases $(65.71 \%)$ and female accounts for 12 cases $(34.29 \%)$. Male: Female ratio in our study was 1.92:1. In our study, headache was the most common presenting complaint in 27 (77.14\%) of cases followed by seizures (51.43\%), focal neurological deficit (31.43\%), vomiting (14.3\%), vertigo $(8.57 \%)$. Other presenting complaints were seen in $5.71 \%$ of cases. Location of the lesions is shown in the Table-1. Out of the 35 patients who were evaluated-majority $16(45.71 \%)$ of them showed size of $>4 \mathrm{~cm}, 12(34.29 \%)$ of them showed lesions of sizes between $2-4 \mathrm{~cm}$ and only in 7 (20\%) lesions size was less than $2 \mathrm{~cm}$. In case of multiple lesions, size of the maximum number of lesions, which were falling in one category was considered. The intensity patterns of cystic lesion based on T1, T2 and FLAIR morphology are shown in the Table-2. Out of the 35 patients who were evaluated -10 (28.57\%) of patients showed diffusion restricting lesions (partial/complete) and 25 (71.43\%) of cases showed no diffusion restriction.

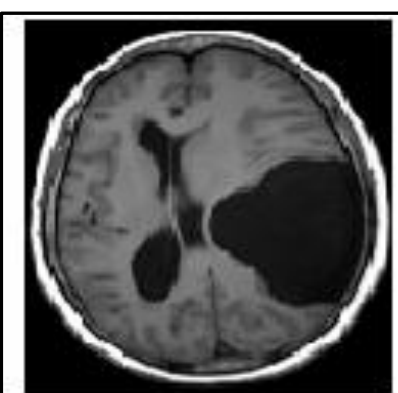

A

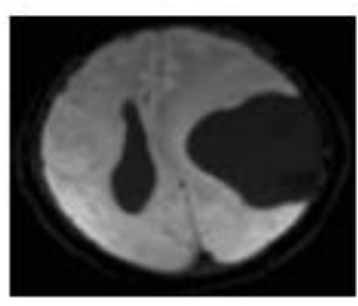

C

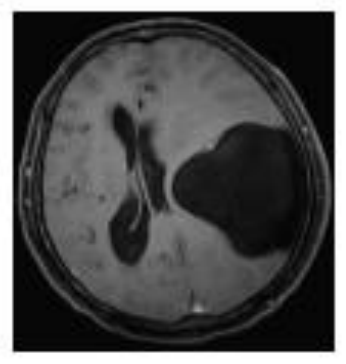

E

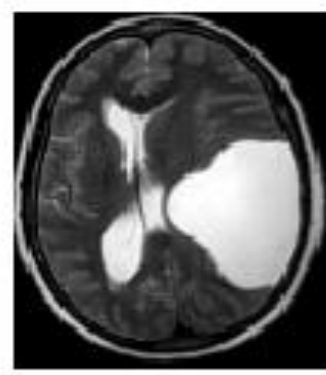

B

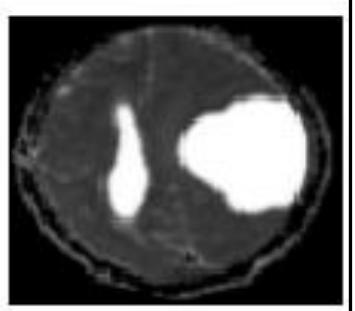

D

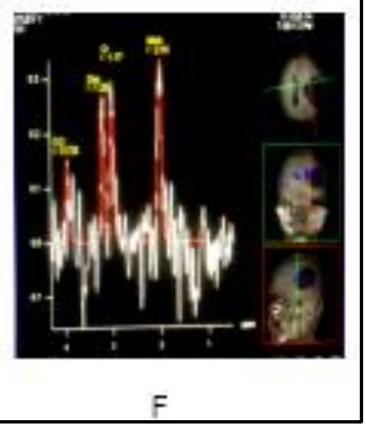

Fig. 1

A 40-year-old female patient. Axial T1WI (A) and axial T2WI (B) show a large extra-axial CSF signal intensity cystic lesion over left temporoparietal convexity. No restriction seen on DWI (C) with high ADC value (D). Axial T1FS postcontrast image (E) shows no significant postcontrast enhancement. (F) MRS shows reduced NAA/Cr ratio with mild lactate peak. Features are suggestive of Arachnoid cyst. 
The postcontrast enhancement pattern of cystic lesions is shown in the Fig 2. Out of total 35 cases evaluated, MRS was possible in 26 cases. Out of 26 cases, 5 (19.23\%) of cases showed noisy spectrum and findings were taken as inconclusive reports and excluded from study.

\begin{tabular}{|l|c|c|}
\hline \multicolumn{1}{|c|}{ Location } & $\begin{array}{c}\text { Number } \\
\text { (n= 35) }\end{array}$ & $\begin{array}{c}\text { Percentage } \\
\text { (\%) }\end{array}$ \\
\hline Intra-axial: & 19 & 54.29 \\
\hline Parenchymal & 14 & 40.00 \\
\hline Intraventricular & 5 & 14.29 \\
\hline Extra-axial: & 16 & 45.71 \\
\hline Midline & 5 & 14.29 \\
\hline Off midline Table 1: Location \\
\hline \multicolumn{2}{|c|}{} \\
\hline
\end{tabular}

\begin{tabular}{|c|c|c|}
\hline $\begin{array}{c}\text { Intensity Patterns of } \\
\text { Cystic Lesion }\end{array}$ & $\begin{array}{c}\text { Number } \\
\text { (n = 35) }\end{array}$ & $\begin{array}{c}\text { Percentage } \\
\text { (\%) }\end{array}$ \\
\hline $\begin{array}{c}\text { T1 CSF like or Low signal } \\
\text { Intensity Pattern }\end{array}$ & 20 & 57.14 \\
\hline $\begin{array}{c}\text { T1 Intermediate signal } \\
\text { Intensity Pattern }\end{array}$ & 10 & 28.57 \\
\hline \multicolumn{2}{|c|}{ T1 High signal } \\
Intensity Pattern & 4 & 11.43 \\
\hline \multicolumn{2}{|c|}{$\begin{array}{c}\text { Table 2: Intensity Patterns of Cystic Lesion } \\
\text { Based On T1, T2 and Flair Morphology }\end{array}$} \\
\hline
\end{tabular}

\section{RESULTS AND OBSERVATIONS}

Spectral quality was interpretable in 21 cases. Out of 21 cases, Reduced NAA/Cr ratio (<1.6) was observed in $18(69.23 \%)$ of cases, Lactate peak in 17 (65.38\%) of cases, Choline peak in 7 (26.92\%) of cases, Increased Cho/Cr ratio ( $>1.5$ ) in 7 (26.92\%) of cases and Lipid peak was observed in $6(23.07 \%)$ of cases. The remaining 1 case (3.84\%) showed NAA peak with increased NAA/Cr ratio. Comparing conventional MRI and 3D CISS for intraventricular cystic lesions, we found out of 4 $(100 \%)$ cases of intraventricular cystic lesions, cyst wall was visualised in $2(50 \%)$ of cases on conventional MRI and in all 4 $(100 \%)$ cases on 3D CISS images. Out of $3(100 \%)$ cases of intraventricular neurocysticercosis, Scolex was visualised only in $1(33.3 \%)$ case on conventional MRI and in all 3 cases $(100 \%)$ on 3D CISS images. Out of the 35 patients who were evaluated, non-neoplastic cystic lesions (Fig 1) were seen in 23 $(65.71 \%)$ of cases, benign neoplastic cystic lesions (Fig 3 ) in 4 $(11.43 \%)$ of cases and malignant neoplastic cystic of cases. (Fig 4) Various cystic lesions of brain diagnosed by MRI in our study are shown in the Table-3. Out of the 35 patients who were evaluated, Arachnoid cyst (20\%) was the most common non-neoplastic cystic lesion, craniopharyngioma (5.7\%) was the most common benign neoplastic cystic lesion and Glioma $(17.1 \%)$ was the most common malignant neoplastic cystic lesion. In the present study, conventional MRI with additional techniques like MRS and 3D CISS together were found to be accurate in $94.28 \%$ of cases (Fig 5). One case of MRI diagnosis of low-grade glioma came out to be high-grade on HPE. One case of MRI diagnosis of cystic astrocytoma came out to be a benign vascular lesion-Haemangioma on HPE.

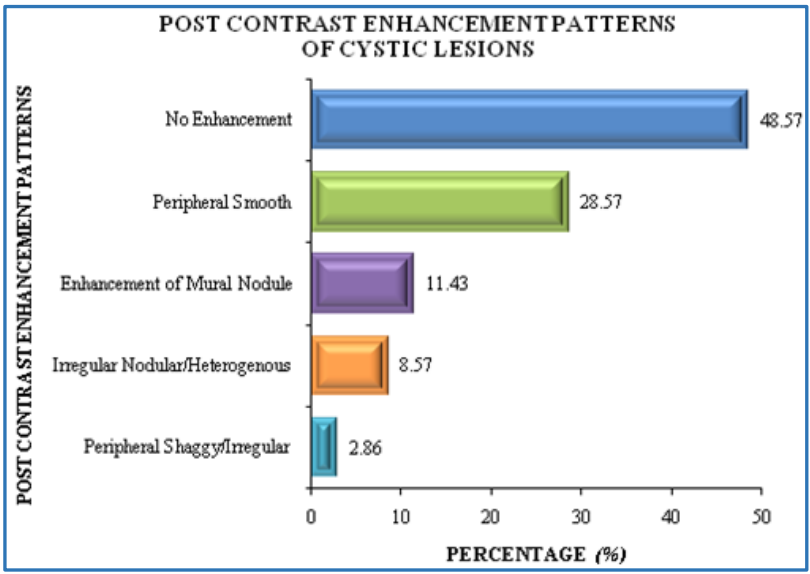

Fig. 2

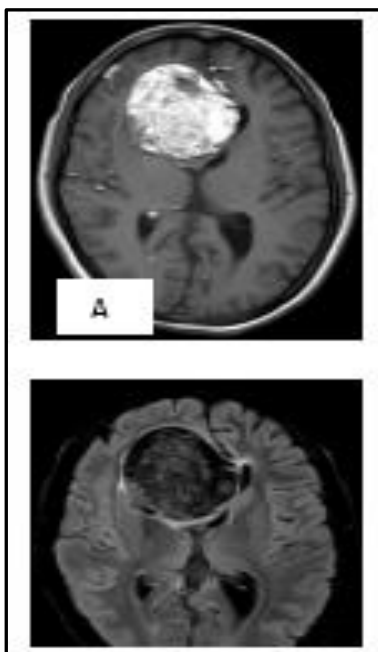

c

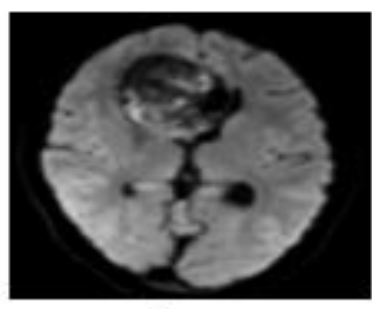

E
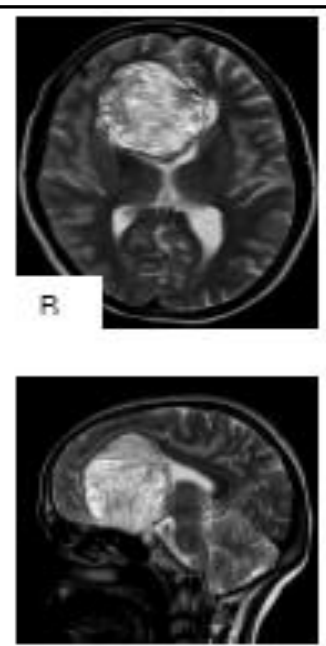

D

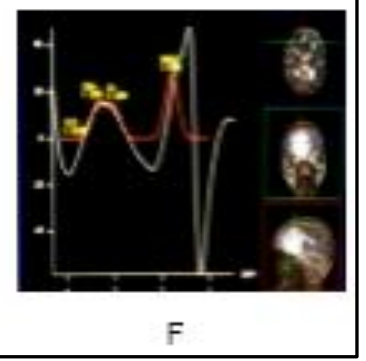

Fig. 3
A 34-year-old female patient. Axial T1WI (A), axial T2WI (B), axial FLAIR (C) and sagittal T2WI (D) images show a large well-defined extra-axial heterogeneous predominantly fat signal intensity cystic lesion in suprasellar and right frontal region in median and right paramedian location. Axial T1WI (A) also shows the multiple tiny hyperintense foci within the sulcal spaces in bilateral cerebral hemispheres suggestive of fat droplets. (E) DWI shows patchy areas of diffusion restriction. (F) MRS showed prominent lip/lactate peak. Features are suggestive of ruptured intracranial dermoid cyst. 


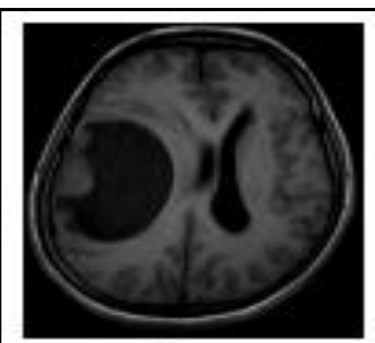

A

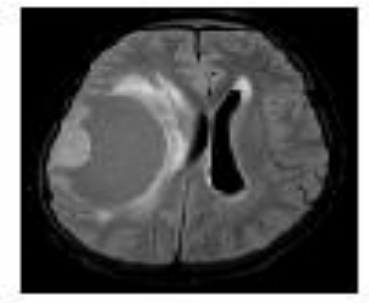

C

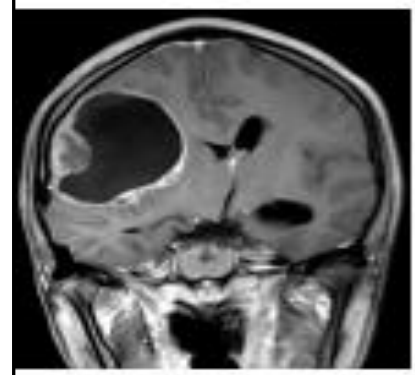

$\equiv$

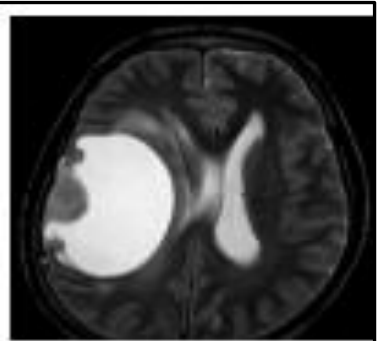

B

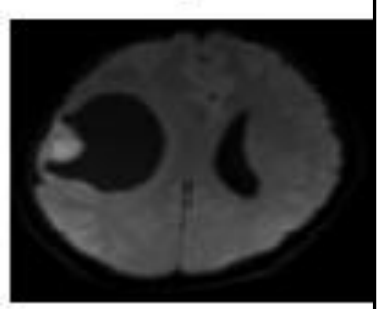

D

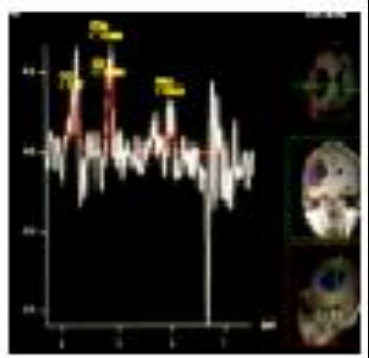

$=$
Fig. 4

A 20-year-old male patient. Axial T1WI (A), axial T2WI (B) and axial FLAIR (C) images show a well-defined intra-axial cystic lesion with eccentric soft tissue mural nodule in right frontoparietal lobe. (D) DWI shows patchy area of restricted diffusion in mural nodule. Coronal T1FS postcontrast image (E) shows enhancement of cyst wall as well as mural nodule. (F) MRS shows choline and lactate peaks, increased $\mathrm{Cho} / \mathrm{Cr}$ ratio and reduced NAA/Cr ratio. Features suggestive of lowgrade glioma.

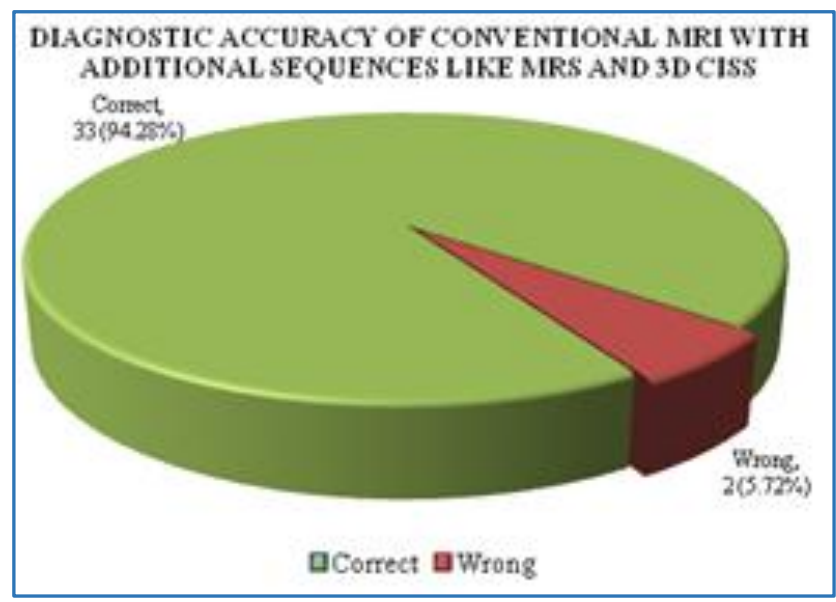

Fig. 5

\section{DISCUSSION}

Magnetic resonance imaging is a non-invasive, multiplanar and highly accurate method with better inherent contrast that demonstrates intracranial cystic lesions accurately.
Conventional MRI with additional techniques like MR spectroscopy and 3D CISS provide an accurate assessment and categorisation in various cystic lesions of brain. Overall, cystic lesions of brain can be categorised into non-neoplastic, benign neoplastic and malignant neoplastic cystic lesions.

\begin{tabular}{|c|c|c|}
\hline Lesions & $\begin{array}{c}\text { Number } \\
\text { (n=35) }\end{array}$ & $\begin{array}{c}\text { Percentage } \\
\text { (\%) }\end{array}$ \\
\hline Arachnoid cyst & 7 & 20.00 \\
\hline Porencephalic cyst & 4 & 11.43 \\
\hline Low-grade glioma & 4 & 11.43 \\
\hline $\begin{array}{c}\text { Intraventricular } \\
\text { NCC }\end{array}$ & 3 & 8.57 \\
\hline High-grade glioma & 2 & 5.71 \\
\hline Epidermoid cyst & 2 & 5.71 \\
\hline $\begin{array}{c}\text { Craniopharyngioma } \\
\text { (Fig 6) }\end{array}$ & 2 & 5.71 \\
\hline $\begin{array}{c}\text { Neuroependymal } \\
\text { cyst }\end{array}$ & 1 & 2.86 \\
\hline Neuroglial cyst & 1 & 2.86 \\
\hline Pineal cyst & 1 & 2.86 \\
\hline Colloid cyst & 1 & 2.86 \\
\hline Dermoid cyst & 1 & 2.86 \\
\hline Cystic metastasis & 1 & 2.86 \\
\hline Cystic schwannoma & 1 & 2.86 \\
\hline DNET & 1 & 2.86 \\
\hline Racemose NCC & 1 & 2.86 \\
\hline Cystic astrocytoma & 1 & 2.86 \\
\hline Rathke's cleft cyst & 1 & 2.86 \\
\hline Total & $\mathbf{3 5}$ & $\mathbf{1 0 0 . 0 0}$ \\
\hline & $\mathbf{T a b l e ~ 3}$ & \\
\hline & & \\
\hline
\end{tabular}

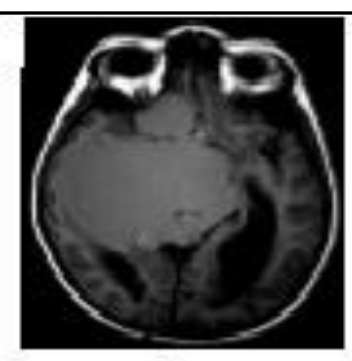

A

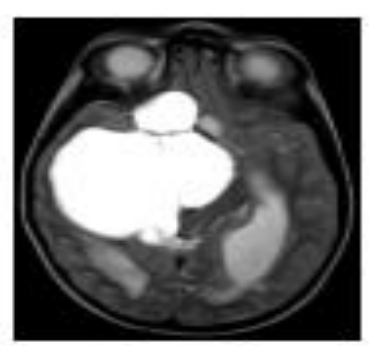

C

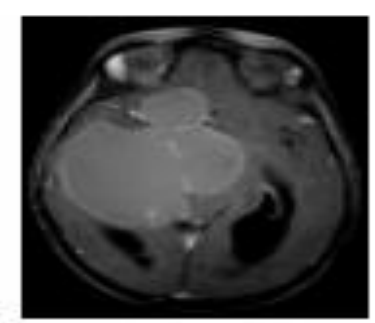

E

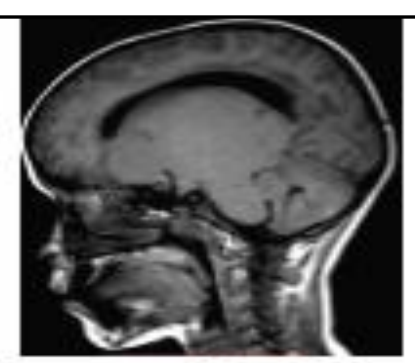

B

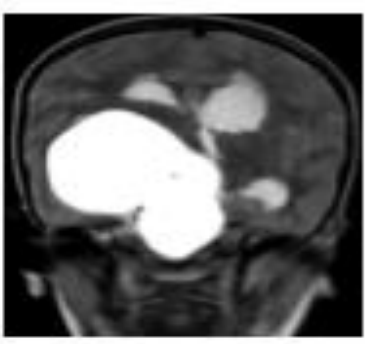

D

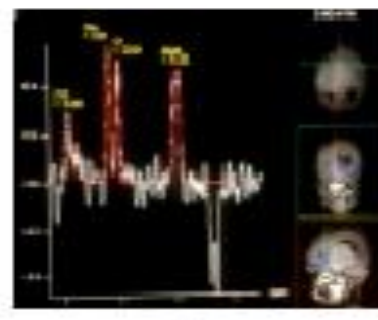

F
Fig. 6 
A 10-year-old male patient. Axial and sagittal T1WI (A and B) and axial and coronal T2WI (C and D) show a large extraaxial lobulated smooth marginated high signal intensity pattern cystic lesion in the suprasellar region with its regional extension. Axial T1FS postcontrast image (E) shows peripheral smooth pattern of contrast enhancement. (F) MRS shows lip/lactate peak with reduced NAA/Cr ratio. Features are suggestive of Craniopharyngioma. (Adamantinomatous type).

Thirty five (35) patients of all age groups were evaluated, the highest incidence of cystic lesions was found in 20-29 years age group accounting for $25.71 \%$ of cases followed by in 30-39 years age group accounting for $20 \%$ of cases and least incidence was observed in age group of $>/=70$ years constituting for $2.86 \%$. Males were 23 (65.7\%) and females 12 (34.3\%). Our findings correlated with study conducted by M. Bergui et al (2001)[6] and N. Meena et al (2015).[5] Headache was the most common presenting complaint in 27 cases (77.14\%), seizures (51.43\%) was the next in frequency followed by focal neurological deficit (FND) (31.43\%), vomiting (14.3\%), vertigo (8.57\%).

Other presenting complaints were seen in $5.71 \%$ of cases. Our findings correlated with study conducted by N. Meena et al (2015)[5] who stated that irrespective of cause the predominant presenting symptom was headache seen in $90 \%$ of the patients followed by seizures (33\%). B. Alami et al (2012) ${ }^{[7]}$ found that arachnoid cyst $(26 \%)$ was the most common benign intracranial cystic lesion followed by porencephalic cyst (14\%). N. Meena et al (2015) ${ }^{[5]}$ found that most common tumours among intracranial space occupying lesions were gliomas (57\%). Our findings were correlated with these studies. Intra-axial lesions were found in 19 (54.24\%) of cases in which $14(40.00 \%)$ lesions were parenchymal and 5 (14.29\%) lesions were intraventricular.

Parietal lobe was the most common location among the intra-axial intraparenchymal group; $4^{\text {th }}$ ventricle was the most common location among intraventricular group of lesions. Of the $16(45.71 \%)$ extra-axial cases, 11 (31.43\%) cases were off midline in location and $5(14.29 \%)$ cases were midline in location. Out of 11 off midline extra-axial lesions, there were 7 cases of arachnoid cysts found in subarachnoid spaces (Fig. 1) and 4 cases, which included 2 cases of epidermoid cysts (Fig. 7 A, B), 1 case of cystic schwannoma and 1 case of racemose NCC found in cisternal spaces.

$\mathrm{CP}$ angle cistern was the most common location among cisternal spaces and temporal convexity was the most common location among subarachnoid spaces. Our findings were in correlation with Osborn AG, et al (2006).[8] Our findings in signal intensity patterns were correlated with studies conducted by Bent O. Kjos et al (1985),[9] Kim Young Joo et al (1997),[10] R.N. Sener (2001) [11] and Maeder PP et al (1990).[12] One case of dermoid cyst showed heterogeneous or mixed signal intensity pattern. Our findings were correlated with Alexandra Opri Gan, et al (2013).[2] Restricted diffusion was in favour of epidermoid cyst. Our findings were correlated with studies conducted by Ahmed Farid Yousef et al (2014).[13] M. Mian et al (2010)[14] and M. Bergui et al (2001).[6]

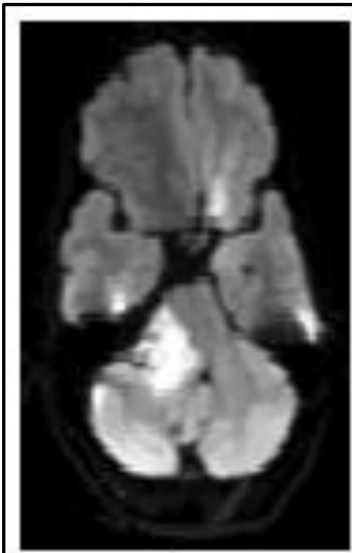

A

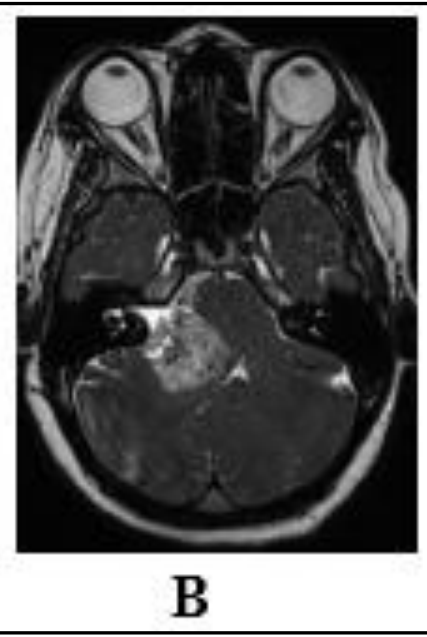

Fig. 7: DWI shows Restricted Diffusion, Epidermoid Cyst (A), (B) Demonstrates the Intracanalicular Extension and IAC Complex IAC, Internal Auditory Canal

Our findings were in correlation with study conducted by Kim Young Joo et al (1997)[10] who also found that no postcontrast enhancement was seen in congenital/developmental non-neoplastic cystic lesions (17 out of 19). Our findings were also correlated with Sophie Taillibert et al (2014).[1] The malignant neoplastic lesions showed irregular nodular/heterogeneous pattern of contrast enhancement. Our findings on NCC were correlated with James G. Smirniotopoulos et al (2007),[15] Srikanth Subbamma Govindappa et al (2000) ${ }^{[16]}$ who had concluded that the 3DCISS sequence was more sensitive and specific than routine SE sequences in the diagnosis of intraventricular cysticercal cysts. Out of 3 cases evaluated, conventional MRI was able to show cyst wall in two cases and scolex in only one case.

3D CISS sequence accurately showed cyst wall as well as scolex in all 3 cases. In our study, $4^{\text {th }}$ ventricle was most common location among 3 cases of intraventricular NCC. Our findings were correlated with studies conducted by Divyata Hingwala et al (2011), [4] Subbamma Govindappa et al (2000),[16] S Kalra et al (2006).[17] We studied 1 (2.86\%) case of neuroependymal cyst in right lateral ventricle. Conventional MRI failed to show the cyst wall and misdiagnosed a case as unilateral lateral ventriculomegaly. Diagnosis of this neuroependymal cyst was only possible after visualisation of cyst wall on 3D CISS images (Fig. 8). Our findings were in correlation with study conducted by Dewen Yang et al (2000).[18] Out of the 35 patients evaluated, spectroscopy was performed and possible in 26 cases and was not performed in 9 cases due to presence of the intralesional calcification, haemorrhage or lesions in close proximity to bone. In our study, on MRS majority, 18 (69.23\%) of cases showed reduced NAA/Cr ratio $(<1.6)$ except 1 case of neuroependymal cyst (Fig 8), 1 case of epidermoid cyst and 1 case of dermoid cyst.

Our findings were correlated with N Meena et al (2015) ${ }^{[5]}$ found that mean NAA/Cr ratio was below normal in all the lesions, lowest values seen in the meningiomas (1.19) and Gliomas (1.03). Lactate was the most common metabolite peak observed accounting for 17 (65.38\%) of cases. Our findings were correlated with study conducted by Kee-Hyun Chang et al (1998) ${ }^{[19]}$ who had concluded that only lactate was commonly observed in a variety of intracranial cystic masses except for abscess and cysticercosis in which resonances of acetate, 
succinate, amino acids and/or unassigned metabolites can be seen in addition to a lactate peak. MRS showed reduced NAA/Cr ratio $(<1.6)$ in arachnoid cysts, porencephalic cysts. Lactate peak was observed in 2 cases.

In 1 case of epidermoid cyst, MRS showed lactate peak with normal NAA/Cr ratio. This correlated with studies conducted by Kee-Hyun Chang et al (1998),[19] A. Shukla-Dave et al (2000)[20] and Harish Poptani et al (1995).[21] Spectroscopy remains an inconclusive diagnostic tool in neuroependymal cyst till the present time; however, resonance of $\mathrm{N}$-acetyl group of compound maybe a subtle indicator of glial tissue within ependymal lining in a case of glioependymal cyst. Malignant neoplastic cystic lesions (primary/secondary) showed choline peak, lactate peak, reduced NAA/Cr ratio and increased Cho/Cr ratio (>1.5). Additionally, lipid peak was observed in 2 cases of high-grade gliomas. Our study correlated with W. Moller-Hartmann et al (2002),[20] Harish Poptani et al (1995),[21] N. Meena et al (2015),[5] Ozan Karatag et al (2010)[22] and Alam M et al (2011).[23] In the present study, conventional MRI with additional techniques like MRS and 3D CISS together were found to be accurate in $94.28 \%$ of cases.

One case of MRI diagnosis of low-grade glioma came out to be high-grade on HPE. One case of MRI diagnosis of cystic astrocytoma came out to be a benign vascular lesionHaemangioma on HPE. The present study correlated with the study conducted by N Meena et al (2015) ${ }^{[5]}$ who found that the diagnostic accuracy of MRS combined with conventional MRI was $94 \%$ keeping histopathology as gold standard. We could not evaluate the statistical significance for 3D CISS in diagnosing the intraventricular cystic lesions in comparison to conventional MRI due to small numbers of intraventricular cystic lesions in our study.

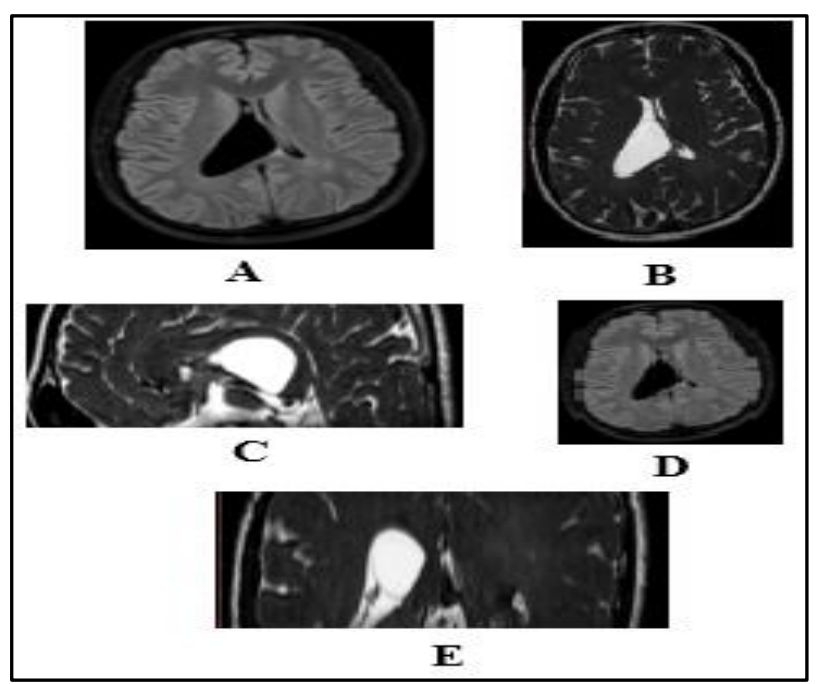

Fig. 8

Axial T1WI (A), axial FLAIR (D) show unilateral right ventricular enlargement. Axial (B), coronal (E) and sagittal (C) 3D CISS images demonstrate a well-defined CSF signal intensity cystic lesion in right lateral ventricle with hypointense cystic wall of intraventricular neuroependymal cyst.

\section{REFERENCES}

1. Taillibert S, Le Rhun E, Chamberlain MC. Intracranial cystic lesions: a review. Curr Neurol Neurosci Rep 2014;14(9):481.
2. Oprisan A, Popescu BO. Intracranial cysts: an imagery diagnostic challenge. The Scientific World, Article ID 172154, 2013;2013:9.

3. Bulakbasi N. Clinical applications of proton MR spectroscopy in the diagnosis of brain tumours. Spectroscopy 2004;18(2):143-53.

4. Hingwala D, Chatterjee S, Kesavadas C, et al. Applications of 3D CISS sequence for problem solving in neuroimaging. Indian J Radiol Imaging 2011;21(2):90-7.

5. Meena N, Bakshi VK, Kumar R, et al. Diagnostic role of magnetic resonance spectroscopy in intracranial space occupying lesions (IC-SOLs): a prospective analytical study. European Society of Radiology 2015:1-32.

6. Bergui M, Zhong J, Bradac GB, et al. Diffusion-weighted images of intracranial cyst-like lesions. Neuroradiology 2001;43(10):824-9.

7. Alami B, Youssef ALM, Addou O, et al. Spectrum of benign intracranial cystic lesions. European Society of Radiology 2012:C-1945.

8. Osborn AG, Preece MT. Intracranial cysts: radiologicpathologic correlation and imaging approach. Radiology 2006;239(3):650-64.

9. Kjos BO, Brant-Zawadzki M, Kucharczyk W, et al. Cystic intracranial lesions: magnetic resonance imaging. Radiology 1985;155(2):363-9.

10. Kim YJ, Young SB, Choi KH, et al. Supratentorial cystic intracranial lesions: MR imaging features. Journal of the Korean Radiological Society 1997;36(1):7-13.

11. Sener RN. Proton MR spectroscopy of craniopharyngiomas. Computerized Medical Imaging and Graphics 2001;25(5):417-22.

12. Maeder PP, Holtås SL, Basibüyük LN, et al. Colloid cysts of the third ventricle: correlation of MR and CT findings with histology and chemical analysis. AJR Am J Roentgenol 1990;155(1):135-41.

13. Yousef AF, Elkharbotly A, Settin M, et al. Role of diffusionweighted MR imaging in discrimination between the intracranial cystic masses. The Egyptian Journal of Radiology and Nuclear Medicine 2014;45(3):869-75.

14. Mian M, Niazi IK, Faruqui ZS, et al. Diffusion-weighted MR imaging in cystic brain lesions. ECR 2015:C-2775.

15. Smirniotopoulos JG, Murphy FM, Rushing EJ, et al. Patterns of contrast enhancement in the brain and meninges. Radiographics 2007;27(2):525-55.

16. Govindappa SS, Narayanan JP, Krishnamoorthy VM, et al. Improved detection of intraventricular cysticercal cysts with the use of three-dimensional constructive interference in steady state MR sequences. AJNR Am J Neuroradiol 2000;21:679-84.

17. Kalra $S$, Jaiswal AK, Behari $S$, et al. Lateral ventricular neurocysticercosis: A case report. Indian J Radiol Imaging 2006;16(4):775-8.

18. Yang D, Korogi Y, Ushio Y, et al. Increased conspicuity of intraventricular lesions revealed by three-dimensional constructive interference in steady state sequences. AJNR Am J Neuroradiol 2000;21:1070-2.

19. Chang KH, Song IC, Kim SH, et al. In vivo single-voxel proton MR spectroscopy in intracranial cystic masses. AJNR Am J Neuroradiol 1998;19:401-5.

20. Moller-Hartmann W, Herminghaus S, Krings T, et al. Clinical application of proton magnetic resonance spectroscopy in the diagnosis of intracranial mass lesions. Neuroradiology 2002;44(5):371-81. 
21. Poptani H, Gupta RK, Roy R, et al. Characterization of intracranial mass lesions with in vivo proton MR spectroscopy. AJNR Am J Neuroradiol 1995;16:1593-603.

22. Karatağ 0, Karatağ GY, Uysal E, et al. Can magnetic resonance spectroscopy adequately differentiate neoplastic from non-neoplastic and low-grade from highgrade lesions in brain masses? Marmara Medical Journal 2010;23(3):326-38.
23. Alam MS, Sajjad Z, Hafeez S, et al. Magnetic resonance spectroscopy in focal brain lesions. J Pak Med Assoc 2011;61(6):540-3. 